\title{
TFW: Annotated Thermal Faces in the Wild Dataset
}

This paper was downloaded from TechRxiv (https://www.techrxiv.org).

\section{LICENSE}

CC BY 4.0

SUBMISSION DATE / POSTED DATE

$13-11-2021 / 17-11-2021$

CITATION

Kuzdeuov, Askat; Aubakirova, Dana; Koishigarina, Darina; Varol, Hüseyin Atakan (2021): TFW: Annotated Thermal Faces in the Wild Dataset. TechRxiv. Preprint. https://doi.org/10.36227/techrxiv.17004538.v1

$\mathrm{DOI}$

10.36227/techrxiv.17004538.v1 


\title{
TFW: Annotated Thermal Faces in the Wild Dataset
}

\author{
Askat Kuzdeuov, Member, IEEE, Dana Aubakirova, Darina Koishigarina, \\ and Huseyin Atakan Varol, Senior Member, IEEE
}

\begin{abstract}
Face detection and localization of facial landmarks are the primary steps in building many face applications in computer vision. Numerous algorithms and benchmark datasets have been proposed to develop accurate face and facial landmark detection models in the visual domain. However, varying illumination conditions still pose challenging problems. Thermal cameras can address this problem because of their operation in longer wavelengths. However, thermal face detection and localization of facial landmarks in the wild condition are overlooked. The main reason is that most of the existing thermal face datasets have been collected in controlled environments. In addition, many of them contain no annotations of face bounding boxes and facial landmarks. In this work, we present a thermal face dataset with manually labeled bounding boxes and facial landmarks to address these problems. The dataset contains 9,202 images of 145 subjects, collected in both controlled and wild conditions. As a baseline, we trained the YOLOv5 [1] object detection model and its adaptation for face detection, YOLO5Face [2], on our dataset. To show the efficacy of our dataset, we evaluated these models on the RWTH-Aachen [3] thermal face dataset in addition to our test set. We have made the dataset, source code, and pre-trained models publicly available at https://github.com/IS2AI/TFW to bolster research in thermal face analysis.
\end{abstract}

Index Terms-Annotated thermal images, thermal faces in the wild, thermal face detection, thermal facial landmark prediction, YOLO, illumination-invariant face detection.

\section{INTRODUCTION}

$\mathbf{F}$ ACE detection is a fundamental step in many computer vision tasks, such as face recognition, age and gender classification, and facial expression analysis. Early face detection algorithms relied on classifiers built on top of feature extractors, such as Haar cascades [4] and Histogram of Oriented Gradients (HOG) [5]. These algorithms are computationally efficient and capable of accurately detecting faces under constrained conditions. However, because of the simplicity of the extracted features, their performance degrades in unconstrained environments. Later, the use of convolutional neural networks (CNNs) for image classification spurred rapid development in face detection [6]. Many annotated face databases and deep learning models have been introduced, significantly improving face detection in the wild [7]. However, variations in head pose, facial expression, occlusion, and especially illumination still pose challenges. The illumination problem is related to the traditional cameras that operate in the visible spectrum. To address this problem, thermal cameras are

A. Kuzdeuov, D. Aubakirova, D. Koishigarina, and H. A. Varol are with the Institute of Smart Systems and Artificial Intelligence, Nazarbayev University, Nur-Sultan City, Kazakhstan. Email: \{askat.kuzdeuov, d.aubakirova, darina.koishigarina, ahvarol\}@ nu.edu.kz

This work has been submitted to the IEEE for possible publication. Copyright may be transferred without notice, after which this version may no longer be accessible.

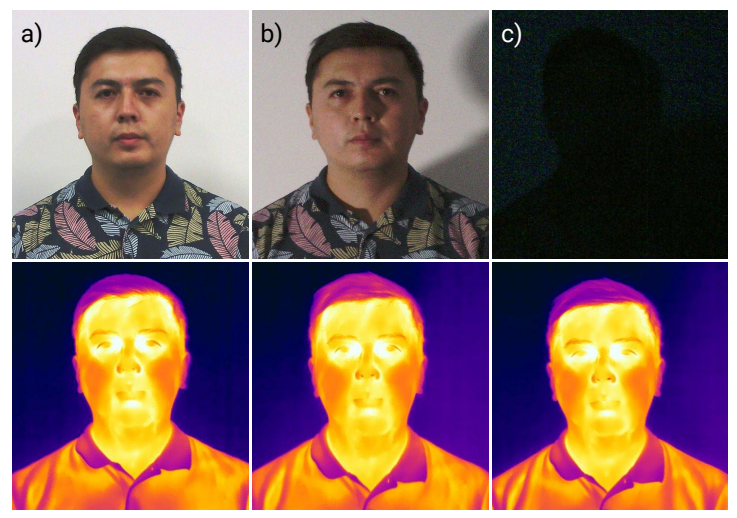

Fig. 1: Examples of visual and thermal images: a) normal illumination, b) low illumination, and c) darkness.

utilized because they are invariant to the illumination and can be used in complete darkness (see Fig. 11).

Nowadays, thermal cameras are employed for a wide range of applications. In biometrics, thermal cameras are used to extract facial physiological patterns for face recognition [8]. The physiological features extracted from thermal faces are also utilized for monitoring vital signs to detect stress [9], deception [10], and affective states [11]. In addition, thermal cameras have gained further popularity after the COVID-19 outbreak for remote fever detection [12]. All of these tasks require robust face detection, especially for monitoring human temperature in public places, such as airports, railway stations, and malls. However, face detection models trained on visual images are not applicable to thermal images because of the modality gap between the domains.

In this regard, many works on thermal face detection have been presented in recent years. For instance, a face detection method based on traditional image processing was introduced in [24]. To test the algorithm, the authors collected 300 images of 30 participants in a controlled environment. The potential of the popular machine learning-based face detection models developed for the visual spectrum was explored in [25]. The researchers trained and evaluated the models on 2,935 thermal images using cross-validation. The results showed that the machine learning-based methods outperform specialized approaches when sufficient training data are available. Deep learning models for thermal face detection have been proposed in several works. In particular, a thermal face detection model based on the R-CNN architecture was trained using transfer learning to examine its performance under various conditions [26], where the original dataset (without augmentation) consisted of 287 images. Transfer learning was applied to train the YOLOv3 object detection model in [27]. The model was 
TABLE I: Summary of thermal face datasets.

\begin{tabular}{|c|c|c|c|c|c|c|c|}
\hline Dataset & Images & Participants & Resolution & Camera & Environment & Bounding box & Landmark \\
\hline VIS-TH [13] & 4,200 & 50 & $160 \times 120$ & FLIR Duo R & controlled & - & - \\
\hline IRIS [14 & 4,228 & 30 & $320 \times 240$ & Raytheon PalmIR Pro & controlled & - & - \\
\hline IRIS-M3 |15 & 2,624 & 82 & $640 \times 480$ & Raytheon PalmIR Pro & controlled & - & - \\
\hline NVIE $[\overline{1} \bar{b} \mid$ & N/A & 215 & $320 \times 240$ & SAT-HY6850 & controlled & - & 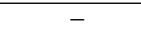 \\
\hline KTFE $[\overline{17}]$ & N/A & 26 & $320 \times 240$ & NEC R300 & controlled & - & - \\
\hline Carl $1 \overline{8}$ & 2,460 & 41 & $160 \times 120$ & TESTO 880-3 & controlled & - & - \\
\hline UL-FMT $\bar{V} \mid \overline{19}]$ & N/A & 238 & $640 \times 512$ & FLIR Phoenix Indigo IR & controlled & - & - \\
\hline SpeakingFaces $|20|$ & $4,581,595$ & 142 & $464 \times 348$ & FLIR T540 & controlled & - & - \\
\hline RWTH-Aachen $|\overline{3}|$ & 2,935 & 90 & $1024 \times 768$ & Infratec HD820 & controlled & - & 68 \\
\hline ARL-VTF 21 & 549,712 & 395 & $640 \times 512$ & FLIR Boson & controlled & $\checkmark$ & 6 \\
\hline OSU Color-Thermal [22] & 17,089 & N/A & $320 \times 240$ & Raytheon PalmIR 250D & wild & - & - \\
\hline TIV $[23$ & 63,782 & N/A & $1024 \times 1024$ & FLIR SC8000 & wild & - & - \\
\hline Our dataset & 9,202 & 145 & $464 \times 348$ & FLIR T540 & controlled, wild & $\checkmark$ & 5 \\
\hline
\end{tabular}

pre-trained on visual images and then fine-tuned using 2,859 thermal images. A comparison of the classical Viola-Jones algorithm with the YOLOv2 and Faster R-CNN object detection models for thermal face detection was presented in [28]. The models were trained on 1,148 manually annotated thermal images, where each image contained a single face. The results showed that Faster R-CNN achieved the highest performance. However, it was the slowest among the considered models, requiring higher computational resources. The YOLOv2 model provided a high detection rate along with faster performance.

The bottleneck in training thermal face detection models is the lack of large labeled datasets specifically collected in wild conditions. For the visual spectrum, there exist many benchmark datasets, such as Face Detection Data Set and Benchmark (FDDB) [29], Multi-Attribute Labelled Faces (MALF) [30], and Wider Face [31] databases. The FDDB dataset contains 2,845 images with 5,171 labeled faces. The MALF dataset consists of 5,250 images and 11,931 labeled faces. The Wider Face dataset has 32,203 images and 393,703 labeled faces with a high degree of variability in terms of scale, occlusion, expression, pose, and illumination, making it the most diverse dataset.

To address this problem, we present the Thermal Faces in the Wild (TFW) dataset with manually labeled bounding boxes and five-point facial landmarks (eye centers, nose tip, and mouth corners). The facial landmarks can assist in exploring unique features in the human face, improving the detection of challenging faces [32]. In addition, the facial landmarks can be used for face alignment, which is a necessary step in face recognition. Several works have addressed the training of thermal facial landmark detection models [33]-[35]. In these works, the models were trained on datasets with a limited number of images collected in controlled environments and annotated manually. The TFW dataset contains 9,202 images and 14,062 labeled faces, of which 5,112 images (5,112 faces) were captured in a controlled environment, while 4,090 images ( 8,950 faces) in wild conditions. The dataset covers challenging scenarios, such as occlusion, head pose variations, different locations, weather conditions, and varying distances from the capturing device. To demonstrate the effectiveness of our dataset, we trained the state-of-the-art YOLOv5 [1] object detection model and its version for face detection YOLO5Face [2] on our dataset and achieved highly accurate performance. We have made the dataset, annotations, source code, and pre-trained models publicly available to facilitate research in thermal face analysis. To the best of our knowledge, our dataset is the first thermal face dataset developed in both controlled and uncontrolled environments and includes facial bounding boxes and landmarks.

The rest of the paper is organized as follows: In Section $\Pi$ we review the existing thermal face datasets. Section [III introduces our dataset including the data collection and annotation steps. In Section IV, we explain the rationale for choosing the YOLOv5 and YOLO5Face models in this work. Section $\mathrm{V}$ provides the details of the training the YOLOv5 and YOLO5Face models and the experimental results. We discuss and interpret the experimental results in Section VI We conclude our work in Section VII

\section{RELATED WORKS}

In this section, we review the existing thermal facial datasets. The characteristics of the datasets are provided in Table I. Most of the datasets have been collected in controlled environments. The VIS-TH dataset [13] contains 50 participants and 4,200 aligned thermal-visual image pairs with a resolution of $160 \times 120$. The dataset was acquired using a FLIR Duo R thermal camera in a controlled environment under various illumination conditions, facial expressions, head poses, and occlusions. The dataset does not provide bounding boxes and facial landmarks. However, it is possible to automatically annotate the thermal images by applying the state-of-the-art face detection and facial landmark prediction models to their visual pairs. Nonetheless, this process may require additional manual calibration [35].

The IRIS [14] and IRIS-M3 [15] datasets were collected in controlled environments using a Raytheon Palm-IR-Pro thermal camera. The IRIS dataset contains 4,228 thermalvisual image pairs $(320 \times 240$ pixels $)$ of 31 participants. The dataset was developed under different lighting conditions, head poses, and facial expressions. The IRIS-M3 dataset has 2,624 thermal-visual image pairs $(640 \times 480$ pixels $)$ of 82 individuals of different ethnicities. The distinguishing feature of the IRISM3 dataset is the presence of natural outdoor illumination. However, the IRIS-M3 dataset does not contain variations in head pose and facial expression. Also, it is important to note that the bounding boxes and facial landmarks are not available 
for both databases. In addition, the visual and thermal images are not aligned, which makes automatic annotation impossible.

The NVIE dataset [16] provides images of 215 individuals with posed and spontaneous facial expressions, such as disgust, fear, surprise, sadness, and anger. The dataset was acquired in a controlled environment under various illumination conditions using a SAT-HY6850 infrared camera. Similarly, the KTFE dataset [17] was designed for emotion recognition. It contains video clips of 26 individuals with seven spontaneous emotions that were recorded in a controlled setting, using a NEC R300 infrared camera. The thermal images in the NVIE and KTFE datasets have a resolution of $320 \times 240$ pixels. The Carl dataset [18] contains 2,460 thermal, visual, and near-infrared images of 41 individuals. The thermal images with a resolution of $160 \times 120$ pixels were collected using a TESTO 880-3 camera in a controlled setting. The UL-MFTV [19] database has images of 238 individuals in the visible, near-infrared, short-wavelength infrared, middlewavelength infrared, and long-wavelength infrared spectra. Moreover, the sessions were held with a time lapse of several years. In addition, the dataset contains images of individuals before and after exposure to cold temperatures or physical activity. SpeakingFaces [20] is the largest database in terms of the number of frames. The dataset provides 4,581,595 thermal-visual image pairs of 142 individuals. The thermal images were acquired using a FLIR T540 thermal camera under various head poses in a controlled environment.

To the best of our knowledge, there are only a few datasets with labeled bounding boxes and facial landmarks. For instance, the RWTH-Aachen dataset [3] has 2,935 images of 90 individuals. The dataset was manually annotated with a 68point landmark set. The images were captured in a controlled environment using an Infratec HD820 camera with a resolution of $1024 \times 768$. The dataset contains various head poses and facial expressions. Bounding boxes and visual pairs are not available. However, bounding boxes can be obtained via the coordinates of facial landmarks. The ARL-VTF dataset [21] contains more than 500,000 thermal-visual image pairs of 395 individuals. The dataset provides bounding boxes and six point landmarks. The images with a resolution of $640 \times 512$ were acquired in a controlled environment.

Most thermal face datasets have been collected in controlled environments, that is an individual sitting at a predefined distance from the camera against a neutral background. Illumination, room temperature, expressions, and poses are predefined. However, in real-world scenarios, the number of individuals per image, background environment, temperature, illumination, scale, and pose vary greatly. One of the few datasets collected under realistic conditions is the OSU ColorThermal Database [22]. The dataset contains 17,089 thermalvisual images of pedestrians captured by a Raytheon PalmIR 250D thermal camera mounted on the side of a building. This dataset is more suitable for human detection and tracking, because faces in the images are too small because of the long distance from the camera. In addition, bounding boxes and facial landmarks are not available. The Thermal Infrared Video (TIV) dataset [23] contains images with higher quality and resolution, thereby allowing the database to be used for
TABLE II: Summary of the TFW dataset.

\begin{tabular}{|r|c|c|c|}
\hline Environment & Participants & Images & Labeled faces \\
\hline Controlled, indoor & 142 & 5,112 & 5,112 \\
\hline Uncontrolled, outdoor & 15 & 4,090 & 8,950 \\
\hline Combined & 145 & 9,202 & 14,062 \\
\hline
\end{tabular}

face detection. However, of the published 63,782 frames, only a subset contains images of humans. Annotations of facial bounding boxes and landmarks are not available either.

In summary, many thermal datasets have been designed for cross-spectral face recognition, anti-spoofing, and emotion recognition. Most of the datasets have been collected in controlled environments. Moreover, only a few of them provide facial bounding boxes and landmarks. The existing datasets collected in wild conditions are not suitable for training face detection models. Therefore, the area of thermal face detection, especially in real-world environments, remains overlooked. Our dataset fills these gaps, by providing thermal images taken in real-world settings, as well as face bounding boxes and facial landmarks.

\section{TFW DATASET}

\section{A. Data Collection}

The TFW dataset contains thermal images acquired in indoor and outdoor environments. The indoor dataset was constructed using our previously published SpeakingFaces dataset [20]. The outdoor dataset was collected using the same FLIR T540 thermal camera with a resolution of $464 \times 348$ pixels, a wave-band of 7.5-14 $\mu \mathrm{m}$, the field of view $24^{\circ}$, and an iron color palette. The dataset was collected with the explicit approval of the Institutional Research Ethics Committee of Nazarbayev University. Each individual participated voluntarily and was informed about the data collection. Also, they were informed that images will be shared as a dataset. Each participant signed a consent form. The characteristics of the TFW dataset are summarized in Table $\amalg$

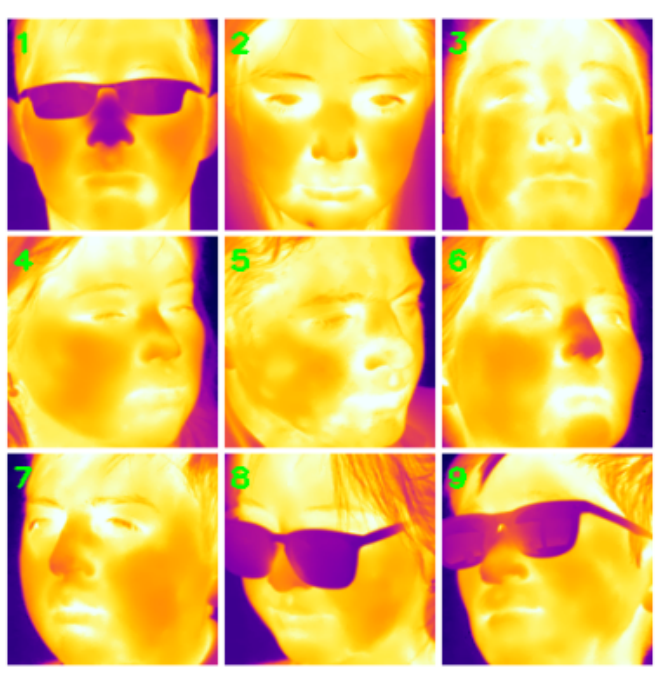

Fig. 2: Examples of cropped thermal facial images from the SpeakingFaces dataset showing nine participants from nine positions. 

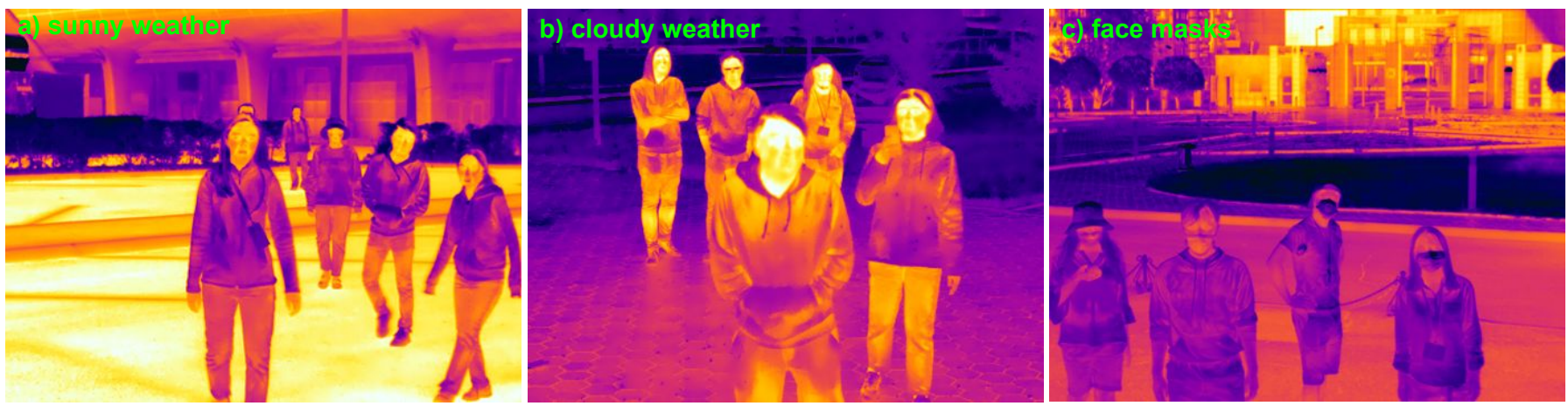

Fig. 3: Thermal faces collected in uncontrolled outdoor environments under different weather conditions and occlusions: a) sunny weather, b) cloudy weather, and c) face masks.

1) Indoor dataset: The SpeakingFaces dataset was collected in a controlled indoor environment. The dataset provides thermal-visual image pairs of 142 individuals, with each participating in two sessions conducted on different days. In each session, the participants were asked to take part in two experiments. In the first experiment, they sat still and silent while the cameras recorded them from nine different positions (see Fig. 2). In the second experiment, the participants read a series of commands displayed on a screen, and their videos were recorded from the aforementioned nine positions. In this work, we extracted one frame from each position to avoid similarities between images. As a result, we obtained 5,112 thermal images (142 participants $\times 2$ sessions $\times 2$ experiments $\times 9$ positions) as shown in Table III Considering that each image contains one participant, the total number of labeled faces is also 5,112 .

2) Outdoor dataset: To collect thermal images in the wild condition, we used outdoor locations on Nazarbayev University campus. The dataset was collected on different days during the summer. We used only outdoor locations since public gatherings in indoor environments were restricted due to the COVID-19 pandemic. Our main goal was to collect images of a randomly walking crowd in public places. Therefore, participants moved in front of the installed thermal camera. They were allowed to walk in any direction within the camera's field of view and make any gestures. The only requirement was to look at the camera as much as possible. We also aimed to collect data in different weather conditions, as the temperature of the environment directly affects thermal images. In addition, different weather conditions provide various natural illumination conditions. Therefore, our dataset contains thermal images taken in sunny and cloudy weather conditions, as shown in Fig. 3. In addition, in some experiments, we asked participants to wear face masks (see Fig. 35) to imitate our new normal due to the pandemic. The total number of unique participants was 15 , where 12 of them had participated in the collection of the SpeakingFaces dataset. In total, we conducted seven sessions at different locations. We kept the first frame and then extracted every 40th frame from each recorded video to avoid similarities between images. We then manually checked the extracted frames to remove similar images and as well as images that did not contain faces. As a result, we obtained

\section{4,090 thermal images.}

\section{B. Data Annotation}

We utilized a graphical image labeling tool [36] to manually annotate bounding boxes and facial landmarks. However, raw thermal images are challenging to annotate because the contours of the eyes and lips are not fully discernible because of the dissipating heat (see Fig. 4a). To address this problem, we processed thermal images before annotation. First, we converted the thermal images from RGB to grayscale. Then, we inverted the grayscale images to obtain negative images. To increase the brightness of the face region, we applied gamma correction to the negative images. By doing so, we improved the visibility of facial features, especially the contours of the eyes and lips (see Fig. 4p). We then added bounding boxes and facial landmarks to the processed images as illustrated in Fig. 4k. The facial landmarks are arranged as follows: 1. the center of the right eye, 2. the center of the left eye, 3. the tip of the nose, 4. the right outer corner of the mouth, and 5. the left outer corner of the mouth. These landmarks are known as primary and play a crucial role in facial identity and face tracking [37]. The same order of landmarks has been used to train many face detection models, such as MTCNN [38], RetinaFace [32], YOLO5Face [2], developed for visual images. The bounding box tightly contains the chin, forehead, and cheek. We annotated all the faces collected in the controlled environment. In the wild dataset, we ignored faces with a high degree of occlusion and of a small scale because of the difficulty of adding five landmarks. The dataset was labeled by four annotators and cross-checked by the main annotator. In total, the TFW dataset contains 145 participants, 9,202 images, and 14,062 labeled faces.

The statistics for the annotated bounding boxes are provided in Table III We classified faces into three categories: small $(<25$ pixels), medium (25-125 pixels), and large $(\geq 125$

TABLE III: Bounding box height statistics for the TFW dataset.

\begin{tabular}{|r|c|c|c|}
\hline Environment & $<25$ pixels & $25-125$ pixels & $\geq 125$ pixels \\
\hline Controlled, indoor & $0 \%$ & $0 \%$ & $100 \%$ \\
\hline Uncontrolled, outdoor & $49.59 \%$ & $50.40 \%$ & $0.01 \%$ \\
\hline Combined & $31.56 \%$ & $32.08 \%$ & $36.36 \%$ \\
\hline
\end{tabular}




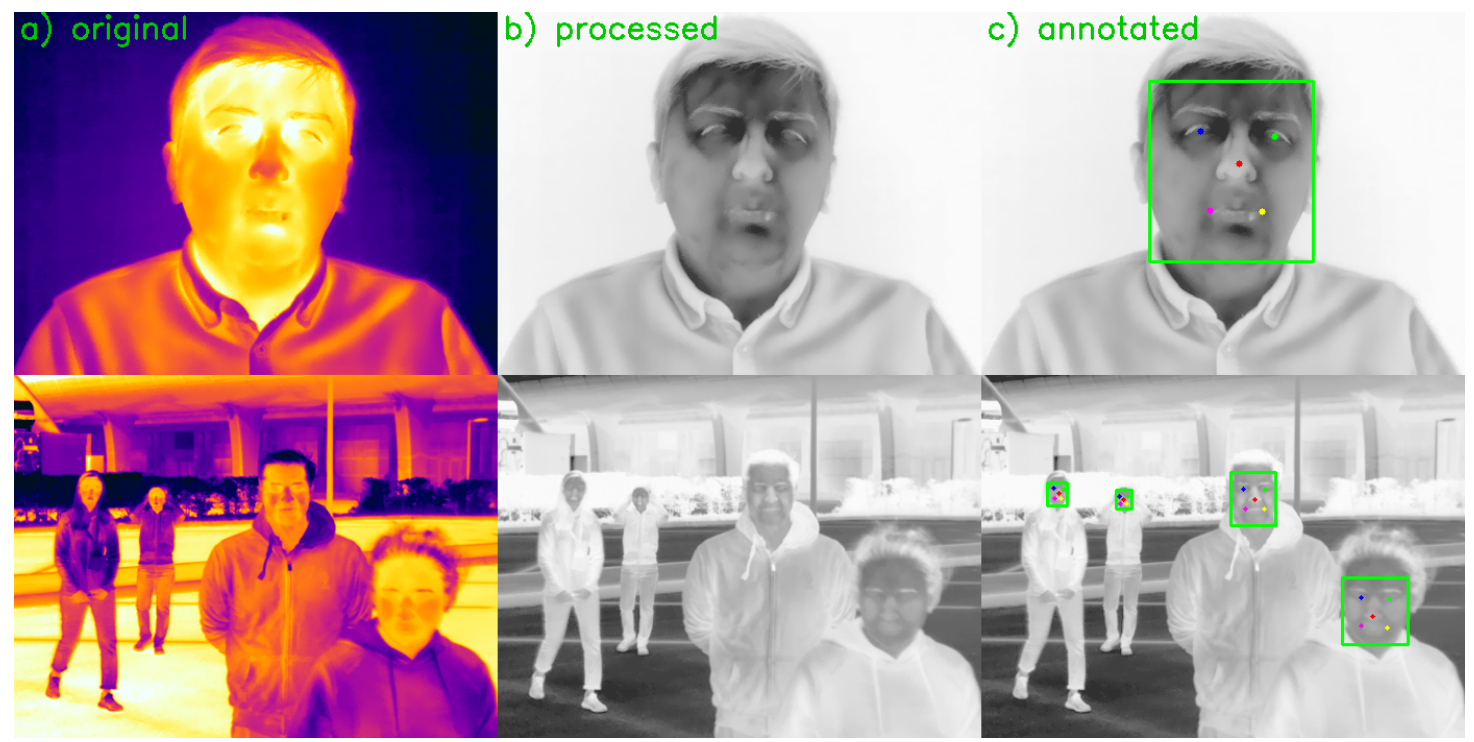

Fig. 4: a) Original thermal image, b) processed thermal image, c) processed thermal image with bounding boxes and facial landmarks. The upper row is in the controlled environment while the lower row is in the wild condition.

pixels) based on their heights. All the faces captured in the controlled environment are large because participants were sitting close to the camera during data collection. The faces captured in the uncontrolled environment were almost evenly distributed between the small and medium categories. Overall, the combined dataset consists of $31.56 \%$ small faces, $32.08 \%$ medium faces, and $36.36 \%$ large faces.

\section{FAce Detection Models}

The state-of-the-art face detection methods leverage the achievements of deep learning-based object detection models. There are two major deep learning-based object detection architectures: one-stage (e.g., SSD [39], YOLO [40], and RetinaNet [41]) and two-stage (RCNN [42], Fast-RCNN [43], and Faster-RCNN [44]). The one-stage models directly predict classes and bounding boxes, while the two-stage models generate the regions of proposals to classify and further refine the bounding boxes of the objects. Therefore, the one-stage detectors are usually faster than the two-stage detectors but are less accurate. This is because the one-stage models tend to have a higher false-positive rate. However, they achieve a higher recall rate than the two-stage models.

In addition, research on face detection has been developing rapidly thanks to the extremely challenging Wider Face benchmark dataset [31].A large number of face detection methods have been proposed to achieve accurate results on this benchmark datase 1] The recent methods include RetinaFace [32], TinaFace [45], MogFace [46], and YOLO5Face [2]. Some of these methods are general object detection models adapted for face detection, while the others explore unique facial features. For instance, the RetinaFace and YOLO5Face face detection architectures use five facial landmarks as an extra supervision signal, while the TinaFace is based on a general object detection model.

${ }_{1}^{1}$ http://shuoyang1213.me/WIDERFACE/WiderFace_Results.html
In this work, we employed the YOLOv5 [1] object detection model and its adapted version for face detection YOLO5Face [2] for the thermal face detection task. We chose these models for several reasons. Firstly, they are relatively faster and have smaller sizes than the other state-of-the-art models, while achieving comparable results on the benchmark datasets. Secondly, they provide models of different sizes, such as nano, small, medium, and large, bringing more options for experiments and finding the best model. In addition, each model can be trained with an additional output block P6 with a stride of 64 to improve the detection of large faces. Thirdly, we investigate the benefits of using facial landmarks as a supervision signal by comparing the results of the YOLOv5 (without facial landmarks) and YOLO5Face (with facial landmarks) models. Finally, the source code for YOLOv5 and YOLO5Face is well documented and publicly available.

The main modifications incorporated into the YOLOv5 model to construct the YOLO5Face model are as follows:

- The regression of five facial landmarks (the center of the right eye, the center of the left eye, the tip of the nose, the right corner of the mouth, the left corner of the mouth) using Wing loss [47] was added to the YOLOv5 network.

- The focus layer was replaced with a Stem-block [48] structure to increase the generalization capability of the network while reducing computational complexity without compromising performance.

- Two light weight models based on the ShuffleNetV2 [49] network were designed in addition to the Cross-StagePartial-connections (CSP) of YOLOv5.

\section{EXPERIMENTS}

\section{A. Splitting the Dataset}

We split our dataset into training, validation, and test sets, as shown in Table IV. The indoor dataset contains images of 142 
TABLE IV: Training, validation, and test set specifications.

\begin{tabular}{|l|c|c|c|c|c|c|}
\hline \multirow{2}{*}{ Dataset } & \multicolumn{2}{|c|}{ Training } & \multicolumn{2}{c|}{ Validation } & \multicolumn{2}{c|}{ Test } \\
\cline { 2 - 7 } & Images & Faces & Images & Faces & Images & Faces \\
\hline Indoor & 3,600 & 3,600 & 432 & 432 & 1,080 & 1,080 \\
\hline Outdoor & 2,958 & 6,664 & 332 & 606 & 800 & 1,680 \\
\hline Total & 6,558 & 10,294 & 764 & 1,038 & 1,880 & 2,760 \\
\hline
\end{tabular}

individuals, where each individual has 36 images. Therefore, we split the indoor dataset based on participants ids as [1-100] subjects (3,600 images, 3,600 faces) for training, [101-130] subjects (1,080 images, 1,080 faces) for testing, and [131142] subjects (432 images, 432 faces) for validation. We split the outdoor dataset based on sessions so that the locations and weather conditions are different in the training, validation, and test sets. Therefore, we assigned the first 2,958 images (6,664 faces) to the training set, the next 332 images (606 faces) to the validation set, and the remaining 800 images (1,680 faces) to the test set. In total, the training set consists of 6,558 images (10,294 faces), the validation set contains 764 images (1,038 faces), and the test set consists of 1,880 images (2,760 faces). In other words, we used $71.3 \%$ of the data for training, $8.3 \%$ for validation, and $20.4 \%$ for testing. In addition to our test set, we evaluated the models on the RWTH-Aachen dataset, which contains 2,935 images (2,935 faces), to demonstrate the efficacy of our dataset. Considering that most of the existing thermal face datasets provide images in grayscale format, including the RWTH-Aachen dataset, we converted our images from the iron color palette to grayscale for training the models.

\section{B. Training Settings}

First, we trained a series of YOLO5Face models of different sizes: nano (YOLOv5n-Face, YOLOv5n6-Face), small (YOLOv5s-Face, YOLOv5s6-Face), medium (YOLOv5mFace, YOLOv5m6-Face), and large-sized models (YOLOv51Face, YOLOv516-Face). As the name suggests, the main difference between these models is the size, - that is the depth (D) and width (W) multiples, and the presence of the P6 output block (see Table V). The YOLOv5n-Face and YOLOv5n6Face models use ShuffleNetv2 as the backbone network, while the other models use CSPNet. The YOLOv5 models were trained on the COCO dataset [50], while the YOLO5Face models were trained on the Wider Face dataset. Both models provide the pre-trained weights. Considering that our dataset is much smaller than the COCO and Wider Face datasets, we applied transfer learning to train the pre-trained models further on our dataset.

We used a single V100 graphics processing unit (GPU) on an NVIDIA DGX2 server to train the nano and small models. We used the default parameters from the original work [2] that achieved state-of-the-art results on the Wider Face dataset, where the batch size and number of epochs were 64 and 250, respectively. To train the medium and large models, we used the Distributed Data Parallel mode in PyTorch to parallelize training on two V100 GPUs, setting the batch size and number of epochs to 32 and 250, respectively. Like in the original paper, we used the SGD optimizer, where the initial and final learning rates were 0.01 and 0.00001 , respectively. The weight decay was set to 0.0005 . The first three epochs were warm-up epochs with a momentum of 0.8. Afterwards, the momentum was changed to 0.937 .

The second model, YOLOv5 also provides nano (YOLOv5n, YOLOv5n6), small (YOLOv5s, YOLOv5s6), medium (YOLOv5m, YOLOv5m6), and large (YOLOv5n, YOLOv5n6) models with and without the P6 output block. All models use the CSPNet as the backbone network. The depth and height multiplies are identical to those of the YOLOv5Face models. We trained the YOLOv5 models as a general object detector that predicts a single class, the face. We used the previous parameters to train the YOLOv5 models to ensure a fair comparison with the YOLO5Face models.

\section{Results}

We evaluated the trained models on the test set of our TFW dataset. We show the results separately for the images collected under the controlled and wild conditions. In this way, we can analyze the capability of models to detect large faces captured in the controlled conditions and small/medium faces captured in the wild. Furthermore, we tested the models on the Aachen dataset to show the generalizability of the models. The models require that the width and height of the input images are divisible by 64 . Considering that the size of images in the dataset is $464 \times 348$, we resized them to $512 \times 384$, while keeping the same aspect ratio. We used the average precision (AP) metric to evaluate the models. To cut off weak predictions, we set a confidence score threshold of $50 \%$ to the output class probability of models. To measure the overlap ratio between the predicted and ground-truth bounding boxes, we used the intersection over union (IoU) metric with a threshold of 0.5 .

The experimental results in Table $\mathrm{V}$ reveal some interesting properties of the models. For instance, all models in YOLOv5 and YOLO5Face achieved an AP score of $100 \%$ on the indoor dataset by successfully detecting all faces. Even though we trained the YOLOv5 models as general object detectors, the results are quite positive. For instance, the YOLOv5n nano model showed AP scores of $92.9 \%$ and $98.3 \%$ on the outdoor and Aachen datasets, respectively. The YOLOv5n6 model with the additional P6 output block further improved the results to 93.3\% and $99.2 \%$ for the outdoor and Aachen datasets, respectively. Similar trends were observed for the small (YOLOv5s), medium (YOLOv5m), and large (YOLOv51) models, where the additional P6 output block provided more accurate results. The YOLOv5m6 medium model with the P6 output block provided the highest accuracy among the YOLOv5 models with an AP score of $94.0 \%$ on the outdoor dataset and an AP score of $99.6 \%$ on the Aachen dataset. The YOLOv5s6 and YOLOv516 models also produced an AP score of $99.6 \%$ on the Aachen dataset, but less accurate results on the outdoor dataset.

The YOLOv5n-Face nano model with the ShuffleNetv2 backbone network achieved an AP score of $92.3 \%$ on the outdoor dataset and an AP score of $99.0 \%$ on the Aachen dataset. The YOLOv5n6-Face with the P6 output block improved the AP score on the Aachen dataset up to $99.4 \%$, 
TABLE V: Results of YOLOv5 and YOLO5Face models on the test set of TFW dataset and on the RWTH-Aachen dataset [3].

\begin{tabular}{|c|c|c|c|c|c|c|c|c|c|c|c|}
\hline \multirow{3}{*}{ Model } & \multirow{3}{*}{ Backbone } & \multirow{3}{*}{$(\mathrm{D}, \mathrm{W})$} & \multicolumn{3}{|c|}{$\mathrm{AP}_{50}$} & \multicolumn{3}{|c|}{ NME } & \multirow{3}{*}{$\begin{array}{l}\text { Speed } \\
\text { V100 } \\
\text { (ms) }\end{array}$} & \multirow{3}{*}{ Params (M) } & \multirow{3}{*}{$\begin{array}{c}\text { Flops }(\mathrm{G}) \\
512 \times 384\end{array}$} \\
\hline & & & \multicolumn{2}{|c|}{ TFW } & \multirow{2}{*}{ Aachen } & \multicolumn{2}{|c|}{ TFW } & \multirow{2}{*}{ Aachen } & & & \\
\hline & & & Indoor & Outdoor & & Indoor & Outdoor & & & & \\
\hline YOLOv5n & CSPNet & $(0.33,0.25)$ & 100 & 92.9 & 98.3 & - & - & - & 6.16 & 1.76 & 0.99 \\
\hline YOLOv5n6 & CSPNet & $(0.33,0.25)$ & 100 & 93.3 & 99.2 & - & - & - & 8.18 & 3.09 & 1.02 \\
\hline YOLOv5s & CSPNet & $(0.33,0.50)$ & 100 & 91.6 & 98.5 & - & - & - & 7.20 & 7.05 & 3.91 \\
\hline YOLOv5s6 & CSPNet & $(0.33,0.50)$ & 100 & 92.6 & 99.6 & - & - & - & 9.05 & 12.31 & 3.88 \\
\hline YOLOv5m & CSPNet & $(0.50,0.75)$ & 100 & 90.9 & 99.1 & - & - & - & 9.59 & 21.04 & 12.07 \\
\hline YOLOv5m6 & CSPNet & $(0.50,0.75)$ & 100 & 94.0 & 99.6 & - & - & - & 12.11 & 35.25 & 11.76 \\
\hline YOLOv51 & CSPNet & $(1.0,1.0)$ & 100 & 91.3 & 98.7 & - & - & - & 12.39 & 46.60 & 27.38 \\
\hline YOLOv516 & CSPNet & $(1.0,1.0)$ & 100 & 92.7 & 99.6 & - & - & $\begin{array}{lll}- & & \end{array}$ & 15.73 & 76.16 & 110.2 \\
\hline YOLOv5n-Face & ShuffleNetv2 & - & 100 & 92.3 & 99.0 & 0.045 & 0.410 & 0.065 & 10.12 & 1.72 & 1.36 \\
\hline YOLOv5n6-Face & ShuffleNetv2 & - & 100 & 91.5 & 99.4 & 0.056 & 0.401 & 0.083 & 13.30 & 2.54 & 1.38 \\
\hline YOLOv5s-Face & CSPNet & $(0.33,0.50)$ & 100 & 92.0 & 99.1 & 0.041 & 0.331 & 0.061 & 8.29 & 7.06 & 3.67 \\
\hline YOLOv5s6-Face & CSPNet & $(0.33,0.50)$ & 100 & 94.1 & 99.6 & 0.047 & 0.379 & 0.069 & 10.86 & 12.37 & 3.75 \\
\hline YOLOv5m-Face & CSPNet & $(0.50,0.75)$ & 100 & 92.2 & 99.4 & $\mathbf{0 . 0 3 9}$ & 0.353 & 0.055 & 11.01 & 21.04 & 11.58 \\
\hline YOLOv5m6-Face & CSPNet & $(0.50,0.75)$ & 100 & 94.4 & 99.6 & 0.043 & 0.362 & 0.062 & 13.97 & 35.45 & 11.84 \\
\hline YOLOv51-Face & CSPNet & $(1.0,1.0)$ & 100 & 91.9 & 99.4 & 0.041 & 0.355 & 0.059 & 13.57 & 46.59 & 25.59 \\
\hline YOLOv516-Face & CSPNet & $(1.0,1.0)$ & 100 & 93.2 & 99.6 & 0.046 & 0.361 & 0.066 & 17.29 & 76.67 & 113.2 \\
\hline
\end{tabular}

but was less accurate $(91.5 \%)$ on the outdoor dataset. As with the YOLOv5 models, using the P6 output block yielded better results on the YOLO5Face models. For instance, the YOLOv5s-Face small model showed AP scores of $92.0 \%$ and $99.1 \%$ on the outdoor and Aachen datasets, respectively. Then, the YOLOv5s6-Face small model with the P6 block improved the AP scores to $94.1 \%$ and $99.4 \%$ for the outdoor and Aachen datasets, respectively. In addition, the YOLOv5m6-Face model improved the AP scores of the YOLOv5m-Face model from 92.2\% and $99.4 \%$ (outdoor) to $94.4 \%$ and $99.6 \%$ (Aachen). Similarly, the YOLOv516-Face large model with the P6 block showed better results than the YOLOv51-Face model.

To measure the performance of the YOLO5Face models in detecting facial landmarks, we used the normalized mean error (NME) metric

$$
N M E=\frac{1}{N} \sum_{i=1}^{N} \frac{\left\|\hat{l}_{i}-l_{i}\right\|}{K \times D_{i}}
$$

where $\left\|\hat{l}_{i}-l_{i}\right\|$ is L2 norm between the coordinates of the ground-truth $\hat{l}_{i}$ and predicted $l_{i}$ facial landmarks, $N$ is the number of detected faces, $K$ is the number of facial landmarks, and $D_{i}$ is the distance between eye centers. The results in Table V reveal interesting insights. For instance, the P6 output block slightly reduces performance in terms of NME, despite providing higher results in terms of AP. The reason might be that the P6 block reduces the feature map size twice, leaving fewer features for regressing the facial landmarks. Moreover, the NMEs on the outdoor dataset are noticeably larger than on the indoor and Aachen datasets. The reason is that the outdoor dataset is much more challenging because of the large variations in scale, pose, and temperature of the environment. In addition, the NMEs on the Aachen dataset are slightly greater than on the indoor dataset. The main reason is that the Aachen dataset is more diverse in terms of facial expressions. Also, we did not use images from the Aachen dataset to train the models.

\section{DISCUSSION}

The experimental results show that the YOLOv5 and YOLO5Face models provide accurate AP scores. However, using facial landmarks as an additional supervision signal yielded higher results. For instance, the YOLOv5s-Face small model improved the AP scores of the YOLOv5s model by $0.4 \%$ and $0.6 \%$ for the outdoor and Aachen datasets, respectively. The YOLOv5s6-Face medium model with the P6 output block is $1.5 \%$ more accurate on the outdoor dataset while having the same accuracy on the Aachen dataset. A similar trend can be seen for the YOLOv5m-Face, YOLOv5m6-Face, YOLOv51-Face, and YOLOv516-Face models. For instance, the YOLOv5m6-Face medium model with the P6 output block improved the AP score of the YOLOv $5 \mathrm{~m} 6$ model by $0.4 \%$ on the outdoor dataset.

The trade-off between the inference speed and accuracy on the outdoor dataset at $0.5 \mathrm{IOU}$ metric is visualized in Fig. 5. The YOLOv5n nano model with $1.76 \mathrm{M}$ parameters is the fastest, processing one image with a resolution of $512 \times 384$ within $6.16 \mathrm{~ms}$. At the same time, the model is more accurate than many larger models. The YOLOv516-Face large model with $113.2 \mathrm{M}$ parameters is the slowest, processing

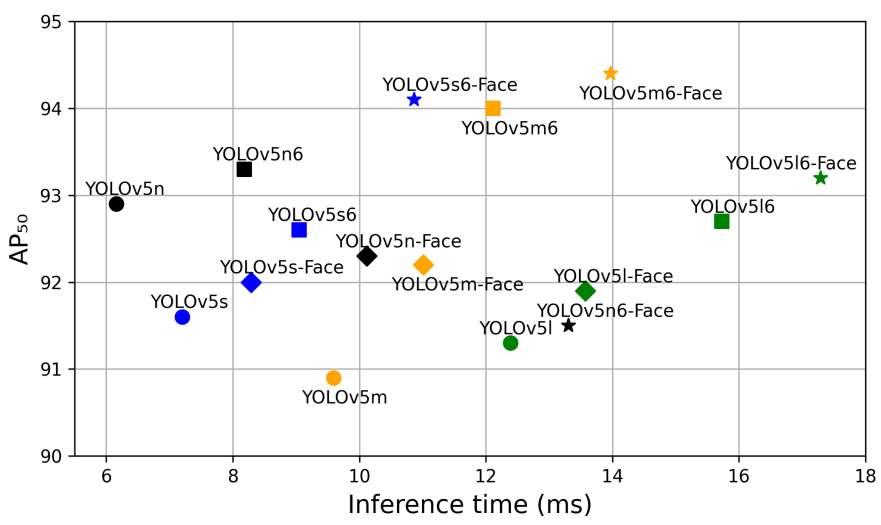

Fig. 5: Trade-off between the speed and accuracy on the AP of the outdoor dataset at $0.5 \mathrm{IOU}$ metric. 


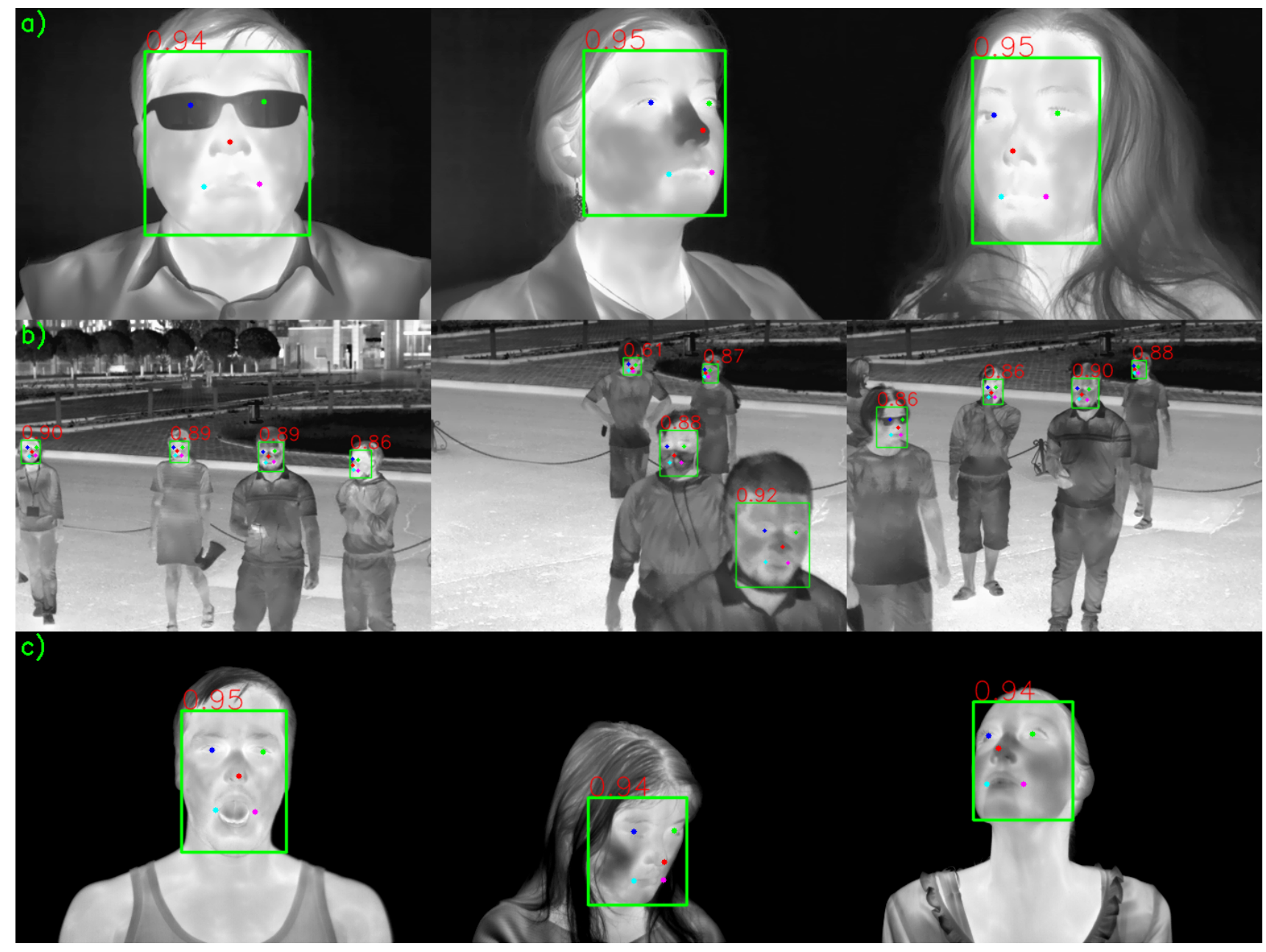

Fig. 6: Examples of the detected bounding boxes, facial landmarks, and corresponding confidence scores obtained via the YOLOv5m6-Face model on randomly selected images from a) TFW (indoor), b) TFW (outdoor), and c) RWTH-Aachen.

one image within $17.29 \mathrm{~ms}$. However, it is less accurate than many smaller models. The inference time of the most accurate YOLOv5m6-Face model is $13.57 \mathrm{~ms}$. The YOLOv5s6-Face model is slightly less accurate than the YOLOv5m6-Face but runs 1.3 times faster.

It is important to note that the accurate results on the Aachen dataset illustrate the efficacy of our dataset. The reason for this is that the Aachen dataset was not used to train and validate the models. Therefore, the models see the Aachen dataset only during testing. In comparison, Kopaczka et al. [25] trained five different machine learning-based face detection algorithms on the Aachen dataset using the leaveone-subset-out cross-validation method. The algorithms considered are the Haar cascade classifier (Viola-Jones (VJ)) [4], Haar cascade classifier with local binary patterns (LBP) [51], HOG [5], deformable parts model (DPM) [52], and pixel intensity comparisons organized in decision trees (PICO) [53]. The performance of the tested algorithms in [25] and our best model YOLOv5m6-Face is given in Table VI The best result in [25] was obtained using the DPM, achieving 1.00 precision and 0.98 recall scores for an IoU of 0.5 . In contrast, our best model, YOLOv5m6-Face, achieves 1.00 precision and 0.99 recall scores on the Aachen dataset for an IoU of 0.5, using only the TFW dataset for training and validation. Moreover, our smallest model, YOLOv5n, provides 0.999 precision and 0.983 recall scores on the Aachen dataset without seeing it during training. We are aware that our deep learning-based models are much more powerful than the older machine learning models. However, we aim to show that our dataset is large and diverse enough to train deep learning models to produce accurate results on an unseen dataset.

The examples of the predicted bounding boxes and facial landmarks on randomly selected images from the test set are shown in Fig. 6. As can be seen from the figure, the model can accurately detect large, medium, and small faces with facial landmarks in the TFW and Aachen datasets. Despite its many advantages, our dataset has some limitations. For instance, the number of unique participants in the wild dataset is much

TABLE VI: Comparing the results in [25] with the results of our best model YOLOv5m6-Face on the Aachen dataset. Precision and recall scores are given for an $\mathrm{IoU}$ of 0.5 .

\begin{tabular}{|r|c|c|c|c|c|c|}
\hline Model & VJ & LBP & HOG & DPM & PICO & YOLOv5m6-Face \\
\hline Precision & 0.93 & 0.97 & 1.00 & 1.00 & 0.90 & $\mathbf{1 . 0 0}$ \\
\hline Recall & 0.98 & 0.92 & 0.93 & 0.98 & 1.00 & $\mathbf{0 . 9 9}$ \\
\hline
\end{tabular}


smaller than in the controlled environment because of the COVID-19 pandemic constraints. In addition, the visual pairs are only available for the images collected in the controlled environment.

\section{CONCLUSION}

Thermal cameras have unique properties, such as insensitivity to illumination, the ability to measure the temperature without contact, and the capability to extract unique physiological patterns from the human face. Therefore, they are gaining popularity in many areas of computer vision, such as face recognition, vital sign recognition, and emotion recognition. Face detection and localization of facial landmarks are the fundamental steps in these tasks. There are many thermal face datasets to train deep learning models. However, most datasets have been collected in controlled environments and provided without facial bounding boxes and landmarks.

To address this problem, we presented a thermal face dataset that contains 9,202 thermal images of 145 individuals. We thoroughly annotated the TFW dataset with facial bounding boxes and five facial landmarks. To the best of our knowledge, this is the first annotated thermal face dataset that provides thermal images collected in both controlled and uncontrolled environments. As a baseline, we trained the YOLOv5 object detection model and its modified version of the YOLO5Face face detection model on our dataset.

The experimental results show that both models achieve high accuracy. However, the use of facial landmarks as an additional supervision signal provided more accurate results. In addition to our test set, we evaluated the models on the Aachen dataset. The results show that the models are generalizable to this dataset as well, highlighting the efficacy of our dataset. However, the accuracy on the outdoor dataset is noticeably lower than on the indoor and Aachen datasets, leaving room for improvement. To reinforce research in thermal face analysis, we have made the source code, dataset, annotations, and pre-trained models publicly available. In our future work, we will focus on developing face applications using our dataset and pre-trained models.

\section{REFERENCES}

[1] G. Jocher. (2020) Yolov5. Last accessed on 2021-08-25:"'https://github. com/ultralytics/yolov5"

[2] D. Qi, W. Tan, Q. Yao, and J. Liu, "YOLO5Face: Why reinventing a face detector," arXiv preprint arXiv:2105.12931, 2021.

[3] M. Kopaczka, R. Kolk, J. Schock, F. Burkhard, and D. Merhof, "A thermal infrared face database with facial landmarks and emotion labels," IEEE Transactions on Instrumentation and Measurement, vol. 68, no. 5, pp. 1389-1401, 2019.

[4] P. Viola and M. Jones, "Rapid object detection using a boosted cascade of simple features," in Proc. of the IEEE Computer Society Conference on Computer Vision and Pattern Recognition (CVPR), vol. 1, 2001, pp. I-I.

[5] N. Dalal and B. Triggs, "Histograms of oriented gradients for human detection," in Proc. of the IEEE Computer Society Conference on Computer Vision and Pattern Recognition (CVPR), vol. 1, 2005, pp. 886-893.

[6] A. Krizhevsky, I. Sutskever, and G. E. Hinton, "ImageNet classification with deep convolutional neural networks," in Advances in Neural Information Processing Systems, F. Pereira, C. J. C. Burges, L. Bottou, and K. Q. Weinberger, Eds., vol. 25. Curran Associates, Inc., 2012. [Online]. Available: https://proceedings.neurips.cc/paper/2012/ file/c399862d3b9d6b76c8436e924a68c45b-Paper.pdf
[7] S. Minaee, P. Luo, Z. Lin, and K. Bowyer, "Going deeper into face detection: A survey," 2021.

[8] P. Buddharaju, I. T. Pavlidis, P. Tsiamyrtzis, and M. Bazakos, "Physiology-based face recognition in the thermal infrared spectrum," IEEE Transactions on Pattern Analysis and Machine Intelligence, vol. 29, no. 4, pp. 613-626, 2007.

[9] S. Kumar, A. S. M. Iftekhar, M. Goebel, T. Bullock et al., "StressNet: Detecting stress in thermal videos," in Proc. of the IEEE Winter Conference on Applications of Computer Vision (WACV), 2021, pp. 9981008.

[10] B. A. Rajoub and R. Zwiggelaar, "Thermal facial analysis for deception detection," IEEE Transactions on Information Forensics and Security, vol. 9, no. 6, pp. 1015-1023, 2014.

[11] B. R. Nhan and T. Chau, "Classifying affective states using thermal infrared imaging of the human face," IEEE Transactions on Biomedical Engineering, vol. 57, no. 4, pp. 979-987, 2010.

[12] D. Perpetuini, C. Filippini, D. Cardone, and A. Merla, "An overview of thermal infrared imaging-based screenings during pandemic emergencies," International Journal of Environmental Research and Public Health, vol. 18, no. 6, 2021. [Online]. Available: https: //www.mdpi.com/1660-4601/18/6/3286

[13] K. Mallat and J.-L. Dugelay, "A benchmark database of visible and thermal paired face images across multiple variations," in Proc. of the International Conference of the Biometrics Special Interest Group (BIOSIG), 2018, pp. 1-5.

[14] B. Abidi, "IRIS thermal/visible face database. IEEE OTCBVS WS series bench," http://vcipl-okstate.org/pbvs/bench/. accessed: 2021-07-02.

[15] H. Chang, H. Harishwaran, M. Yi, A. Koschan, B. Abidi, and M. Abidi, "An indoor and outdoor, multimodal, multispectral and multi-illuminant database for face recognition," in Proc. of the Conference on Computer Vision and Pattern Recognition (CVPR), 2006, pp. 54-54.

[16] S. Wang, Z. Liu, S. Lv, Y. Lv et al., "A natural visible and infrared facial expression database for expression recognition and emotion inference," IEEE Transactions on Multimedia, vol. 12, no. 7, pp. 682-691, 2010.

[17] H. Nguyen, K. Kotani, F. Chen, and B. Le, "A thermal facial emotion database and its analysis," in Image and Video Technology, R. Klette, M. Rivera, and S. Satoh, Eds. Springer, Berlin Heidelberg, 2014, pp. 397-408.

[18] V. Espinosa-Duró, M. Faundez-Zanuy, and J. Mekyska, "A new face database simultaneously acquired in visible, near-infrared and thermal spectrums," Cognitive Computation, vol. 5, pp. 119-135, 032013.

[19] R. Ghiass, H. Bendada, and X. Maldague, "Université Laval face motion and time-lapse video database (UL-FMTV)," 012018.

[20] M. Abdrakhmanova, A. Kuzdeuov, S. Jarju, Y. Khassanov, M. Lewis, and H. A. Varol, "SpeakingFaces: A large-scale multimodal dataset of voice commands with visual and thermal video streams," Sensors, vol. 21, no. 10, 2021. [Online]. Available: https://www.mdpi.com/ $1424-8220 / 21 / 10 / 3465$

[21] D. Poster, M. Thielke, R. Nguyen, S. Rajaraman et al., "A largescale, time-synchronized visible and thermal face dataset," in Proc. of the IEEE/CVF Winter Conference on Applications of Computer Vision (WACV), 2021, pp. 1559-1568.

[22] J. W. Davis and V. Sharma, "Background-subtraction using contourbased fusion of thermal and visible imagery," Computer Vision and Image Understanding, vol. 106, no. 2, pp. 162-182, 2007. [Online]. Available: https://www.sciencedirect.com/science/article/pii/ S1077314206001834

[23] Z. Wu, N. Fuller, D. Theriault, and M. Betke, "A thermal infrared video benchmark for visual analysis," in Proc. of the IEEE Conference on Computer Vision and Pattern Recognition (CVPR), 2014, pp. 201-208.

[24] Y. K. Cheong, V. V. Yap, and H. Nisar, "A novel face detection algorithm using thermal imaging," in Proc. of the IEEE Symposium on Computer Applications and Industrial Electronics (ISCAIE), 2014, pp. 208-213.

[25] M. Kopaczka, J. Nestler, and D. Merhof, "Face detection in thermal infrared images: A comparison of algorithm-and machine-learning-based approaches," in Proc. of the International Conference on Advanced Concepts for Intelligent Vision Systems, 2017, pp. 518-529.

[26] T. Vuković, R. Petrović, M. Pavlović, and S. Stanković, "Thermal image degradation influence on R-CNN face detection performance," in Proc. of the Telecommunications Forum (TELFOR), 2019, pp. 1-4.

[27] D. Li, X. Zhu, X. Chen, D. Tian, X. Hu, and G. Qin, "Thermal imaging face detection based on transfer learning," in Proc. of the International Conference on Smart Grid and Electrical Automation (ICSGEA), 2021, pp. 263-266.

[28] M. Kowalski, A. Grudzień, and W. Ciurapiński, "Detection of human faces in thermal infrared images," Metrology and Measurement Systems, vol. $28,2021$. 
[29] V. Jain and E. Learned-Miller, "FDDB: A benchmark for face detection in unconstrained settings," University of Massachusetts, Amherst, Tech. Rep. UM-CS-2010-009, 2010.

[30] B. Yang, J. Yan, Z. Lei, and S. Z. Li, "Fine-grained evaluation on face detection in the wild," in Proc. of the IEEE International Conference on Automatic Face and Gesture Recognition, vol. 1, 2015, pp. 1-7.

[31] S. Yang, P. Luo, C. C. Loy, and X. Tang, "WIDER FACE: A face detection benchmark," in Proc. of the IEEE Conference on Computer Vision and Pattern Recognition (CVPR), 2016.

[32] J. Deng, J. Guo, E. Ververas, I. Kotsia, and S. Zafeiriou, "RetinaFace: Single-shot multi-level face localisation in the wild," in Proc. of the IEEE/CVF Conference on Computer Vision and Pattern Recognition (CVPR), 2020, pp. 5202-5211.

[33] M. Kopaczka, K. Acar, and D. Merhof, "Robust facial landmark detection and face tracking in thermal infrared images using active appearance models," in Proc. of the International Conference on Computer Vision Theory and Applications (VISAPP), 2016, pp. 150-158.

[34] W.-T. Chu and Y.-H. Liu, "Thermal facial landmark detection by deep multi-task learning," in Proc. of the IEEE International Workshop on Multimedia Signal Processing (MMSP), 2019, pp. 1-6.

[35] J. Keong, X. Dong, Z. Jin, K. Mallat, and J.-L. Dugelay, "Multi-spectral facial landmark detection," in Proc. of the IEEE International Workshop on Information Forensics and Security (WIFS), 2020, pp. 1-6.

[36] K. Wada, "labelme: Image Polygonal Annotation with Python," https: //github.com/wkentaro/labelme 2016.

[37] O. Celiktutan, S. Ulukaya, and B. Sankur, "A comparative study of face landmarking techniques," EURASIP Journal on Image and Video Processing, vol. 2013, pp. 1-27, 2013.

[38] K. Zhang, Z. Zhang, Z. Li, and Y. Qiao, "Joint face detection and alignment using multitask cascaded convolutional networks," IEEE Signal Processing Letters, vol. 23, no. 10, pp. 1499-1503, 2016.

[39] W. Liu, D. Anguelov, D. Erhan, C. Szegedy, S. Reed, C.-Y. Fu, and A. C. Berg, "SSD: Single shot multibox detector," in Computer Vision ECCV 2016, B. Leibe, J. Matas, N. Sebe, and M. Welling, Eds. Cham: Springer International Publishing, 2016, pp. 21-37.

[40] J. Redmon, S. Divvala, R. Girshick, and A. Farhadi, "You Only Look Once: Unified, real-time object detection," in Proc. of the IEEE Conference on Computer Vision and Pattern Recognition (CVPR), 2016, pp. 779-788.

[41] T.-Y. Lin, P. Goyal, R. Girshick, K. He, and P. Dollár, "Focal loss for dense object detection," in Proc. of the IEEE International Conference on Computer Vision (ICCV), 2017, pp. 2999-3007.

[42] R. Girshick, J. Donahue, T. Darrell, and J. Malik, "Rich feature hierarchies for accurate object detection and semantic segmentation," in Proc. of the IEEE Conference on Computer Vision and Pattern Recognition (CVPR), 2014, pp. 580-587.

[43] R. Girshick, "Fast R-CNN," in Proc. of the IEEE International Conference on Computer Vision (ICCV), 2015, pp. 1440-1448.

[44] S. Ren, K. He, R. Girshick, and J. Sun, "Faster R-CNN: Towards realtime object detection with region proposal networks," IEEE Transactions on Pattern Analysis and Machine Intelligence, vol. 39, no. 6, pp. 11371149,2017

[45] Y. Zhu, H. Cai, S. Zhang, C. Wang, and Y. Xiong, "TinaFace: Strong but simple baseline for face detection," ArXiv, vol. abs/2011.13183, 2020.

[46] Y. Liu, F. Wang, B. Sun, and H. Li, "MogFace: Rethinking scale augmentation on the face detector," ArXiv, vol. abs/2103.11139, 2021.

[47] Z.-H. Feng, J. Kittler, M. Awais, P. Huber, and X.-J. Wu, "Wing loss for robust facial landmark localisation with convolutional neural networks," in Proc. of the IEEE Conference on Computer Vision and Pattern Recognition, 2018, pp. 2235-2245.

[48] R. J. Wang, X. Li, and C. X. Ling, "Pelee: A real-time object detection system on mobile devices," arXiv preprint arXiv:1804.06882, 2018.

[49] N. Ma, X. Zhang, H.-T. Zheng, and J. Sun, "ShuffleNet V2: Practical guidelines for efficient CNN architecture design," in Proc. of the European Conference on Computer Vision (ECCV), September 2018.

[50] T.-Y. Lin, M. Maire, S. Belongie, L. Bourdev, R. Girshick et al., "Microsoft COCO: Common objects in context," 2015.

[51] S. Liao, X. Zhu, Z. Lei, L. Zhang, and S. Z. Li, "Learning multiscale block local binary patterns for face recognition," in Advances in Biometrics, S.-W. Lee and S. Z. Li, Eds. Berlin, Heidelberg: Springer Berlin Heidelberg, 2007, pp. 828-837.

[52] P. F. Felzenszwalb, R. B. Girshick, D. McAllester, and D. Ramanan, "Object detection with discriminatively trained part-based models," IEEE Transactions on Pattern Analysis and Machine Intelligence, vol. 32, no. 9, pp. 1627-1645, 2010.
[53] N. Markuš, M. Frljak, I. S. Pandžić, J. Ahlberg, and R. Forchheimer, "Object detection with pixel intensity comparisons organized in decision trees," 2014

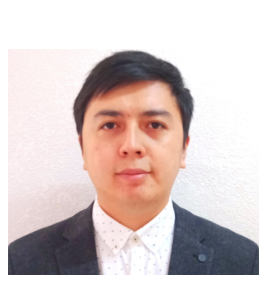

Askat Kuzdeuov received his B.S. in automation and control engineering from Almaty University of Power Engineering and Telecommunication, Kazakhstan, in 2014. From 2014 to 2019, he was a software engineer in the oil \& gas industry, participating in more than thirty local and international projects. In 2019, he received M.S. in robotics from Nazarbayev University, Nur-Sultan, Kazakhstan. He is currently a data scientist with the Institute of Smart Systems and Artificial Intelligence (ISSAI) at Nazarbayev University. His research interests include computer vision, machine learning, and robotics.

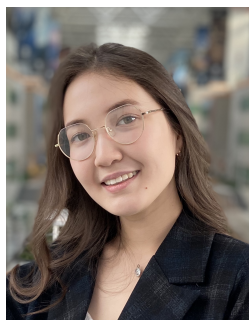

Dana Aubakirova is a senior student in mathematics with a focus on machine learning and statistics at Nazarbayev University. Currently, she is a research assistant at the Institute of Smart Systems and Artificial Intelligence (ISSAI) in Nazarbayev University focusing on thermal face and facial landmark detection. Her research interests include computer vision, deep learning, and human-robot interaction.

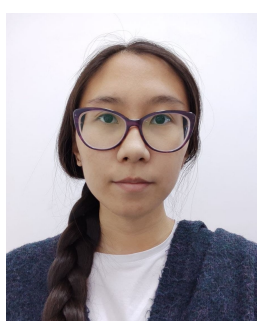

Darina Koishigarina is an undergraduate student in computer science at Nazarbayev University, NurSultan, Kazakhstan. She is currently a research assistant at the Institute of Smart Systems and Artificial Intelligence (ISSAI) at Nazarbayev University. Her research interests are computer vision, deep learning, and statistical learning.

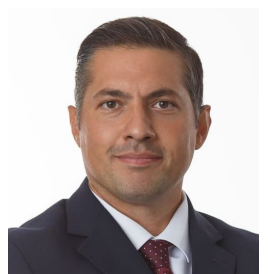

Huseyin Atakan Varol (M'09, SM'16) received his B.S. degree in mechatronics engineering from Sabanci University, Istanbul, Turkey, in 2005, and M.S. and Ph.D. degrees in electrical engineering from Vanderbilt University, Nashville, TN, USA, in 2007 and 2009, respectively. He was a postdoctoral research associate and then a research assistant professor at Vanderbilt University from 2009 to 2011. In 2011, he joined the faculty of Nazarbayev University, Nur-Sultan, Kazakhstan, where he currently chairs the Department of Robotics and Mechatronics and directs the Institute of Smart Systems and Artificial Intelligence (ISSAI). His research interests include soft robotics, machine learning, computer vision, sensor fusion, and tensegrity. He has published over 80 technical papers on related topics in international journals and conferences.

Dr. Varol was a finalist for the KUKA Innovation Award in 2014. He was also the recipient of the IEEE International Conference on Rehabilitation Robotics Best Paper Award in 2009, and the IEEE Engineering in Medicine and Biology Society Outstanding Paper Award in 2013. He serves as a Technical Editor of the IEEE/ASME Transactions on Mechatronics. 